\title{
A Study on Solving the Linear Seepage Flow Model with $r_{\text {we }}$ of Composite Reservoir
}

\author{
Y. Wang, X.X. Dong, S.C. Li, H.E. Li \\ School of Science, Xihua University \\ Chengdu, China
}

\author{
D.D. Gui \\ Beijing Dongrunke Petroleum Technology Co., Ltd \\ Beijing, China
}

\begin{abstract}
This paper studies the linear seepage flow model of composite reservoir under three different kinds of outer boundary conditions (infinite boundary, constant pressure boundary and closed boundary) which consider the well-bore storage and introducing the effective radius. On the basic of the theory of similar structure of solution of the BVP of differential equation, this paper obtains solutions of dimensionless reservoir pressure and dimensionless bottom-hole pressure of composite reservoir in Laplace space. Solutions of dimensionless reservoir pressure and dimensionless bottom-hole pressure have similar structure. Similar structures of solutions are convenient for analyzing the influence of reservoir parameters on reservoir pressure and bottom-hole pressure. The study supplements the other studies on composite reservoir.
\end{abstract}

Keywords-composite reservoir; boundary value problem (BVP); seepage flow; similar structure of the solution; similar kernel function

\section{INTRODUCTION}

Composite reservoir is the reservoir that involves two regions with different properties (the rock-oriented one and the fluid-oriented one), namely, the inner region and the outer one which are separated by a discontinuous interface. There have been many foreign and domestic researches in the area of composite reservoir.

Reference1 built seepage flow models of composite reservoir. Also it studied pressure drop, transient behavior and transient pressure analysis of composite reservoir. Reference 2 studied the equation of pressure diffusion in radically composite reservoirs by the application of Green function, Fourier transform and Laplace transform and has given a general method to solve the class of equations. Since seepage flow models of composite reservoir usually can be transformed into BVPs of composite modified Bessel equation, so Reference 3 studied the BVP of second-order composite linear homogeneous composite modified Bessel equation. It studied the similar structure of the solution and put forward a new method for solving this class boundary value problem. References [4-6] studied the fractal composite reservoir under three kinds of outer boundary conditions (infinite boundary, constant pressure boundary and closed boundary) which consider the well-bore storage and the skin factor. Solutions of the reservoir pressure and bottom-hole pressure in Laplace space were obtained by using the Laplace transform. A similar structure of the solution was discovered by analyzing the percolation characteristics of fractal composite reservoir under three different kinds of outer boundary conditions. References $[7,8]$ obtained the similar structure of the solution of dimensionless reservoir pressure and dimensionless bottom-hole pressure of the composite reservoir under the three different kinds of outer boundary conditions (infinite boundary, constant pressure boundary and closed boundary). Furthermore, they made the theoretical graph and analyzed the impact of the well-bore storage and skin factor on dimensionless reservoir pressure and dimensionless bottom-hole pressure by using the numerical inversion. References [9-11] had studied the well test analysis models of the composite reservoirs, fractal composite reservoir and multi-bed composite reservoirs in which the well-bore storage effect and the skin effect are taken into consideration.

Based on the above researches, this paper studied the linear seepage flow model of composite reservoir which considers the well-bore storage and introduces the effective radius. By using Laplace transformation and introducing dimensionless variables, the linear seepage flow model is transformed into a boundary value problem of composite modified zero-order Bessel equation. Based on the theory of the similar structure of the solution of the boundary value problem of differential equation, this paper obtains solutions of dimensionless reservoir pressure and dimensionless bottom-hole pressure of composite reservoir in Laplace space. The study lays the foundation for studying the seepage flow of composite reservoir and supplements the study on composite reservoir.

\section{THE LiNEAR SEEPAGE FlOW MODEL WITH $r_{\text {we }}$ OF COMPOSITE RESERVOIR}

Here we studied the linear seepage flow model of composite reservoir which the well-bore storage and the effective radius were taken into consideration. To formulate the percolation model, the main assumptions are as follows: law;

1) The single-phase micro compressible fluid obeys Darcy's

2) The reservoir has equal thickness, each direction is horizontal and has a same nature;

3) The capillary single-phase horizontal flow without gravity effect;

4) The well production quantity is constant;

5) There is no additional pressure drop existing on the interface between the two seepage regions; 
6) Formation pressure is initial reservoir pressure $p_{0}$ before producing.

The linear seepage flow basic equations of composite reservoir which consider the well-bore storage and the effective radius are as follows.

Inter region:

$$
\frac{\partial^{2} p_{1}}{\partial r^{2}}+\frac{1}{r} \frac{\partial p_{1}}{\partial r}=\frac{1}{\eta_{1}} \frac{\partial p_{1}}{\partial t} \quad r_{\omega}<r<\alpha r_{\omega}, t>0
$$

Outer region:

$\frac{\partial^{2} p_{2}}{\partial r^{2}}+\frac{1}{r} \frac{\partial p_{2}}{\partial r}=\frac{1}{\eta_{2}} \frac{\partial p_{2}}{\partial t} \quad \alpha r_{\omega} \leq r \leq \beta r_{\omega}, t>0$

Initial condition:

$$
p_{1}(r, 0)=p_{2}(r, 0)=p_{0}
$$

Inner boundary condition:

$$
\left\{\begin{array}{l}
p_{\omega}(t)=p_{1}\left(r_{\omega e}, t\right) \\
\left.\left(r \frac{\partial p_{1}}{\partial r}\right)\right|_{r=r_{\rho e}}=\frac{\mu_{1}}{2 \pi k_{1} h}\left(B q+C \frac{d p_{\omega}}{d t}\right)
\end{array}\right.
$$

Convergence condition:

$$
\left\{\begin{array}{l}
p_{1}\left(\alpha r_{\omega}, t\right)=p_{2}\left(\alpha r_{\omega}, t\right) \\
\left.\frac{k_{1}}{\mu_{1}} \frac{\partial p_{1}}{\partial r}\right|_{r=\alpha r_{\omega}}=\left.\frac{k_{2}}{\mu_{2}} \frac{\partial p_{2}}{\partial r}\right|_{r=\alpha r_{\omega}}
\end{array}\right.
$$

Outer condition:

$$
p_{2}(R, t)=p_{0}, \text { or }\left.\quad \frac{\partial p_{2}}{\partial r}\right|_{r=R}=0 \text {, or }\left.\quad p_{2}(r, t)\right|_{r=R \rightarrow \infty}=p_{0}
$$

III. The Linear Seepage Flow Model of Composite RESERVOIR IN LAPLACE SPACE

In order to facilitate the research and description, firstly, dimensionless variables are introduced:

$$
\begin{gathered}
P_{i D}\left(r_{D}, t_{D}\right)=\frac{2 \pi k_{1} h}{B q \mu_{1}}\left[p_{0}-p_{i}(r, t)\right] \quad(i=1,2) \\
t_{D}=\frac{k_{1} t}{\phi_{1} \mu_{1} C_{t_{1}} r_{\omega}^{2}}, \quad T_{D}=\frac{t_{D}}{C_{D}} \quad r_{D}=\frac{r}{r_{\omega} e^{-S}}, R_{D}=\frac{R}{r_{\omega} e^{-S}}, \\
C_{D}=\frac{C}{2 \pi \phi_{1} C_{t_{1}} h r_{\omega}^{2}}, \sigma=\frac{\eta_{1}}{\eta_{2}}=\frac{\phi_{2} \mu_{2} C_{t_{2}}}{\phi_{1} \mu_{1} C_{t_{1}}} \cdot \frac{k_{1}}{k_{2}}, \lambda=\left(\frac{k_{2}}{\mu_{2}}\right) /\left(\frac{k_{1}}{\mu_{1}}\right)
\end{gathered}
$$

Secondly, the Laplace transform is taken to the linear seepage flow model of composite reservoir with dimensionless variable $_{D}$, i.e.

$$
\begin{gathered}
\bar{P}_{i D}\left(r_{D}, z\right)=\int_{0}^{\infty} e^{-z t_{D}} P_{i D}\left(r_{D}, t_{D}\right) d t_{D} \quad(i=1,2), \\
\bar{P}_{\omega D}(z)=\int_{0}^{\infty} e^{-z t_{D}} P_{\omega D}\left(t_{D}\right) d t_{D} .
\end{gathered}
$$

Finally, the BVP of the ODE with parameter $Z$ (where $Z$ is Laplace space variable) is obtained as below:

$$
\left\{\begin{array}{l}
\frac{d^{2} \bar{P}_{1 D}}{d r_{D}^{2}}+\frac{1}{r_{D}} \cdot \frac{d \bar{P}_{1 D}}{d r_{D}}=\frac{z}{C_{D} e^{-2 S}} \bar{P}_{1 D}, \quad 1 \leq r_{D} \leq \alpha \\
\frac{d^{2} \bar{P}_{2 D}}{d r_{D}^{2}}+\frac{1}{r_{D}} \cdot \frac{d \bar{P}_{2 D}}{d r_{D}}=\frac{\sigma z}{C_{D} e^{-2 S}} \bar{P}_{2 D}, \quad r_{D}>\alpha \\
\bar{P}_{\omega D}(z)=\bar{P}_{1 D}(1, z) \\
\left(r_{D} \frac{d \bar{P}_{1 D}}{d r_{D}}\right)_{r_{D}=1}=-\bar{q}_{D}(z)+z \bar{P}_{\omega D} \\
\bar{P}_{1 D}(\alpha, z)=\bar{P}_{2 D}(\alpha, z),\left.\quad \frac{d \bar{P}_{1 D}}{d r_{D}}\right|_{r_{D}=\alpha}=\left.\lambda \frac{d \bar{P}_{2 D}}{d r_{D}}\right|_{r_{D}=\alpha} \\
\bar{P}_{2 D}(\infty, z)=0 \text { or } \bar{P}_{2 D}\left(R_{D}, z\right)=0 \text { or }\left.\frac{d \bar{P}_{2 D}}{d r_{D}}\right|_{r_{D}=R_{D}}=0
\end{array}\right.
$$

IV. Solving The Linear Seepage Flow Model of COMPOSITE RESERVOIR

Solutions of two regions of the BVP (7) which have the form of the product of continued fraction (the similar structure) are obtained as follows.

$$
\bar{P}_{1 D}\left(r_{D}, z\right)=\bar{q}_{D}(z) \cdot \frac{1}{z+\frac{1}{-\frac{2}{z}+\Phi_{1}(1, z)}} \cdot \frac{1}{-\frac{2}{z}+\Phi_{1}(1, z)} \cdot \Phi_{1}\left(r_{D}, z\right) \quad\left(1 \leq r_{D} \leq \alpha\right)
$$




$$
\begin{aligned}
& \bar{P}_{2 D}\left(r_{D}, z\right)=\bar{q}_{D}(z) \cdot \frac{1}{z+\frac{1}{-\frac{2}{z}+\Phi_{1}(1, z)}} \cdot \frac{1}{-\frac{2}{z}+\Phi_{1}(1, z)} \\
& \cdot \frac{\Psi_{0,1}\left(\alpha, \alpha, \sqrt{\frac{z}{C_{D} e^{-2 S}}}\right)}{\Phi_{2}(\alpha, z) \sqrt{\frac{z}{C_{D} e^{-2 S}}} \Psi_{1,1}\left(1, \alpha, \sqrt{\frac{z}{C_{D} e^{-2 S}}}\right)+\lambda \Psi_{1,0}\left(1, \alpha, \sqrt{\frac{z}{C_{D} e^{-2 S}}}\right)} \cdot \Phi_{2}\left(r_{D}, z\right)\left(r_{D}>\beta\right)
\end{aligned}
$$

where the similar kernel function of outer region is delimited as below:

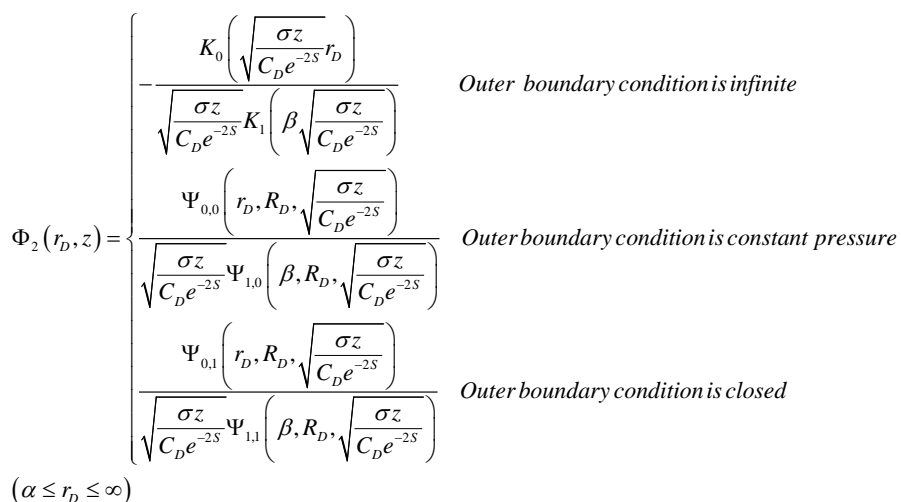
below.

The similar kernel function of inter region is delimited as

$$
\begin{aligned}
& \int \frac{\lambda \Psi_{0,0}\left(r_{D}, \alpha, \sqrt{\frac{z}{C_{D} e^{-2 S}}}\right)+\sqrt{\frac{z}{C_{D} e^{-2 S}}} \Psi_{0,1}\left(r_{D}, \alpha, \sqrt{\frac{z}{C_{D} e^{-2 S}}}\right) \Phi_{21}(\alpha, z)}{\lambda \sqrt{\frac{z}{C_{D} e^{-2 S}}} \Psi_{1,0}(1, \alpha, \sqrt{z})+\frac{z}{C_{D} e^{-2 S}} \Psi_{1,1}\left(1, \alpha, \sqrt{\frac{z}{C_{D} e^{-2 S}}}\right) \Phi_{21}(\alpha, z)} \\
& \Phi_{1}\left(r_{D}, z\right)=\left\{\begin{array}{l}
\frac{\lambda \Psi_{0,0}\left(r_{D}, \alpha, \sqrt{\frac{z}{C_{D} e^{-2 S}}}\right)+\sqrt{\frac{z}{C_{D} e^{-2 S}}} \Psi_{0,1}\left(r_{D}, \alpha, \sqrt{\frac{z}{C_{D} e^{-2 S}}}\right) \Phi_{22}(\alpha, z)}{\lambda \sqrt{\frac{z}{C_{D} e^{-2 S}}} \Psi_{1,0}\left(1, \alpha, \sqrt{\frac{z}{C_{D} e^{-2 S}}}\right)+\frac{z}{C_{D} e^{-2 S}} \Psi_{1,1}\left(1, \alpha, \sqrt{\frac{z}{C_{D} e^{-2 S}}}\right) \Phi_{22}(\alpha, z)}
\end{array}\right. \\
& \text { - Outer boundary condition is constant pressure } \\
& \lambda \Psi_{0,0}\left(r_{D}, \alpha, \sqrt{\frac{z}{C_{D} e^{-2 S}}}\right)+\sqrt{\frac{z}{C_{D} e^{-2 S}}} \Psi_{0,1}\left(r_{D}, \alpha, \sqrt{\frac{z}{C_{D} e^{-2 S}}}\right) \Phi_{23}(\alpha, z) \\
& \begin{array}{c}
\lambda \sqrt{\frac{z}{C_{D} e^{-2 S}}} \Psi_{1,0}\left(1, \alpha, \sqrt{\frac{z}{C_{D} e^{-2 S}}}\right)+\frac{z}{C_{D} e^{-2 S}} \Psi_{1,1}\left(1, \alpha, \sqrt{\frac{z}{C_{D} e^{-2 S}}}\right) \Phi_{23}(\alpha, z) \\
\text {-Outer boundary condition is closed }
\end{array} \\
& \left(1 \leq r_{D} \leq \alpha\right)
\end{aligned}
$$

where

$$
\Psi_{m, n}(\alpha, \beta, y)=I_{m}(\alpha y) K_{n}(\beta y)+(-1)^{m-n+1} K_{m}(\alpha y) I_{n}(\beta y)
$$

and $I_{n}(\cdot), K_{n}(\cdot)$ are respectively the first and the second class of modified Bessel functions of order $n$ [12].

\section{SYMBOL DESCRIPTION}

The symbol meanings are listed below.

$B$-Formation volume factor (dimension);

$C_{t}$-Compressibility $\left(\mathrm{MPa}^{-1}\right)$;
$C$-Well-bore storage coefficient $\left(\mathrm{m}^{3} / \mathrm{MPa}\right)$;

$k$-Permeability $\left(\mu \mathrm{m}^{2}\right)$;

$p$-Reservoir pressure ( $\mathrm{MPa}$ );

$p_{\omega}$-Well-bore pressure ( $\mathrm{MPa}$ );

$q$-Production rate or injection rate $\left(\mathrm{m}^{3} / \mathrm{d}\right)$;

$\phi$-Porosity (dimensionless);

$\mathrm{R}$ - The outer boundary radius ( $m$ );

$r$-Well-bore radius $(m)$;

$r_{\text {we }}$-Effective well-bore radius, $(\mathrm{m})$;

$S$ — Skin factor (dimensionless);

$\mu$-Viscosity $(M P a \cdot s)$;

$p_{0}$ - Initial reservoir pressure $(M P a)$;

$\boldsymbol{h}$-Reservoir thickness ( $m$ );

Z -Laplace space variable;

$\eta$-Pressure transmitting coefficient $\left(\mu m^{2} \cdot M P a\right)$;

$\boldsymbol{t}$-Time $(h)$;

-_Laplace domain;

D_Dimensionless;

w-Well-bore parameter

\section{CONCLUSIONS}

Based on the reasoning mentioned above, the following conclusions can be drawn.

1) By using Laplace transformation and introducing dimensionless variables, the linear seepage flow model of composite reservoir is transformed into a BVP of composite zero-order modified Bessel equation. Solutions of dimensionless reservoir pressure and dimensionless bottom-hole pressure are obtained and they have similar structures. Base on the continued fractions Equations (8)-(10), we can directly analyze the effect of the well-bore storage and effective radius on the reservoir pressure and bottom hole pressure.

2) The linear seepage flow model of composite reservoir has the similar structure of solutions under three different outer boundary conditions. The difference is that similar kernel functions are different under three different outer boundary conditions. 


\section{ACKNOWLEDGMENT}

This work is supported the by the Scientific Research Fund of the Sichuan Provincial Education Department of China

(Grant No. 12ZA164).

\section{REFERENCES}

[1] D. Peon, AOSTRA, H. Chhina, AEC. Society of Petroleum Engineers. 1989, 5: 28-31.

[2] Fikri J, Kuchuk, Tarek M, Habashy. Transport in Porous Media. 19(1995): 199-232.

[3] P.H Liu , Z.C. Chen, S.C. Li. Journal of Xihua University (Natural Science Edition), 2006, 25(2):23-26.

[4] S.C. Li, T.P. Zheng. Journal of Jilin University (Engineering and Technology Edition), 2004, 34:104-107.

[5] X.R. Deng, S.C. Li. Journal of Xihua University (Natural Science Edition), 2005, 24(2):4-7.

[6] C.X. Xu, S.C. Li, W.B. Zhu. Drilling \& Production Technology, 2006, 29(5):39-42.

[7] P.S. Zheng, S.C. Li, W.Z. Xu. Drilling \& Production Technology, 2007, 30(3): 49-50(-62).

[8] S.C. Li, P.S. Zheng, Y.F. Zhang. Journal of Mathematics in Practice and Theory, 2008, 38(3):23-28.

[9] Z.G. Yang, Y.X. Chen, X. Wang. Petrochemical Industry Application, 2011,30(3):16-19.

[10] J.X. Luo, L.H. Zhang, Y.L. Zhao, et al. Journal of Yangtze University (Nature Science Edition), 2011,8(2):65-67.

[11] F.X. Zhang, B.C. Wang, Y.T. Fei, et al. Journal of Southwest Petroleum University (Science \& Technology Edition), 2010,32(4):99-102.

[12] Liu Shishi, Liu Shida. Special Function. Beijing: China Meteorological Press, 2002. 